Sharif University of Technology
Scientia Iranica
SCIENTIA

\title{
A less-effort method for spatial network planning
}

\author{
M.R. Habibi ${ }^{\mathrm{a}}$, M. Rashidinejad ${ }^{\mathrm{b}, *}$, A. Hajebrahimi ${ }^{\mathrm{a}}$, and A. Abdollahi ${ }^{\mathrm{b}}$ \\ a. Department of Electrical Engineering, Kerman Graduate University of Technology, Kerman, Iran. \\ b. Department of Electrical Engineering, Shahid Bahonar University of Kerman, Kerman, Iran.
}

Received 23 June 2015; received in revised form 2 June 2016; accepted 29 October 2016

\section{KEYWORDS}

Geographical

Information System

(GIS);

Spatial network

planning;

Transmission

expansion planning;

Transmission line

routing.

\begin{abstract}
This paper presents a method to solve Spatial Network Planning (SNP) in power systems expansion. SNP is an integration of Transmission Expansion Planning (TEP) and optimal line routing problems. In fact, SNP considers the impacts of lines routing on the network power flow. Therefore, it can exploit such impacts to attain a significant techno-economic solution. In this work, a preprocessing stage is employed to reduce the computational effort demanded by line routing. Furthermore, a meta-heuristic algorithm is engaged to solve such a complicated problem. Applying these modifications made it possible to solve SNP for large-scale systems with high-resolution rasterized maps. The proposed method is applied to a 3-bus system, an 18-bus test system as well as the original and modified versions of the IRAN southeast high-voltage transmission network to demonstrate the capability of the proposed methodology.
\end{abstract}

(C) 2018 Sharif University of Technology. All rights reserved.

\section{Introduction}

Transmission Expansion Planning (TEP) is an essential issue in the development of power systems. TEP problem offers the most economic expansion plan for transmission lines, while this plan must be capable of facilitating the forecasted future load as well as future generation patterns [1-3]. A comprehensive review of this problem is provided in [4].

A route of a transmission line has a significant influence on line reactance as well as construction costs. These are two important parameters in a TEP concerning power flow study. In recent years, the optimal line routing problem has been investigated to find the most economical route for a power line $[5,6]$,

*. Corresponding author.

E-mail addresses: m.habibi@student.kgut.ac.ir (M.R.

Habibi); mrashidi@mail.uk.ac.ir (M. Rashidinejad);

ahajebrahimi@ieee.org (A. Hajebrahimi);

a.abdollahi@uk.ac.ir (A. Abdollahi) where a general model for overhead line routing is presented in [7]. This model uses the satellite images to identify environmental constraints while evaluating transmission line investment. In fact, the model employs the developing image-processing methods for such an application. West et al. [8] presents a distribution line routing method for rural areas, where it divides the studied area into different cost regions. The practical obstacles are modeled as infinite cost regions and they are modified in [9] using Geographical Information System (GIS). In fact, GIS is a proper tool for transmission line routing $[10,11]$. In the early literature of power line routing, vector GIS maps are employed to describe environmental elements. A vector map describes multifarious regions and infrastructures by irregular points, lines and polygons, which impose some difficulties on optimal line routing problem [12]. Reference [13] describes a method to convert a GIS vector map into a rasterized map which is a regular grid of cells where each cell represents properties of a geographical position. GIS rasterized map is adopted in $[5,6,12]$ as a state-of-the-art model for GIS mapping. 
Other recent studies are focused on more accurate cost evaluation of line routes. Summarily, most researchers in this field are trying to optimize the equipment installation and maintenance costs subject to geographic, environmental, social, and legal constraints [6].

In the most traditional view, the optimal routes of candidate lines are specified before solving TEP. Therefore, the construction costs and their reactance are kept as constants [14-18]. However, the interaction of TEP with optimal routing of these lines is considered in three different studies as follows:

Interaction with substation placing problem. An interaction between these two problems is modeled in the studies dealing with simultaneous TEP and substation placing [19]. In this case, the route of new lines connected to unconstructed substations should be evaluated during the optimization process and cannot be determined before solving TEP.

Interaction with power-line technology selection. One model of TEP presented in [20] refers to the dependency of line routing on technology selection of new lines. For example, the optimal route of a line is influenced by the decision of constructing overhead lines or underground cables as well as using HVAC or HVDC technology.

The Spatial Network Planning (SNP). This model of TEP problem is presented in [12]. SNP considers a modification of the candidate line routes as an option to influence network power flows. This may help diminish power flow violations and lead to the expansion that is more economical [12]. Shu et al. [21] presents SNP considering generation expansion planning. In [22] some other aspects of power system expansion such as substation placement and sizing, as well as the line type selection, are added to SNP problem while it considers operation costs and uses an $\mathrm{AC}$ power flow.

The SNP is solved in [12] via a very complex nonlinear programming formulation. Although $[12,21,22]$ illustrate a good framework for SNP problem, the main drawback of such a formulation is that the number of variables and constraints will increase dramatically especially when a high-resolution rasterized map is considered. Therefore, the computational complexities as well as computational effort of such modeling may restrict its usage only for poor-resolution GIS rasterized maps.

Here, a method is proposed to solve the most basic model of SNP problem (i.e. presented in [12]). It employs a preprocessing stage to reduce the computational effort required to handle line routing subproblems. The output of this pre-processing stage illustrates the optimal construction costs of each circuit as a function of its desired reactance. Using such a preprocessing stage is indispensable to handling power line routing sub-problems; otherwise, the evaluation of a single-trial solution may take hours in a system with high-resolution rasterized maps. Moreover, a metaheuristic method has been developed to solve such a complicated problem.

The rest of this paper is organized as follows: In Section 2, the proposed SNP mathematical modeling is introduced. Section 3 describes the proposed methodology to provide line routing preprocessed data. The developed solution algorithm for SNP problem is presented in Sections 4. Numerical case studies are provided in Section 5. Finally, Section 6 is devoted to concluding remarks.

\section{Mathematical modeling problem}

Here, a new formulation for Spatial Network Planning (SNP) problem has been developed. The SNP is an integration of TEP and optimal line routing, while its goal is to find the optimal network expansion plan as well as the optimal route of new lines in order to satisfy the operation constraints with less investment costs. The operational constraints are formulated based on DC Power Flow (DCPF) model. A reformulation is also performed to facilitate the model to be able to employ pre-processed data.

\subsection{SNP mathematical framework}

Jun et al. [12] proposed the objective function of SNP as:

$$
\begin{gathered}
v=\min \Sigma_{k=1}^{N_{c}} \Sigma_{p \in M} \Sigma_{\dot{p} \in N(p)} 0.5 \mu_{k}(p, p ́ p) D(p, \dot{p}) B_{k}(p) \\
N(p)=\left\{\dot{p} \mid \sqrt{(X(p)-X(\dot{p}))^{2}+\left(Y(p)-Y\left(p^{\prime}\right)\right)^{2}}\right. \\
\leq \sqrt{2} w, p \neq \dot{p}\}
\end{gathered}
$$

Therefore, 8 neighbours for each cell can be defined. The binary variables $\mu_{k}\left(p, p^{\prime}\right)$ (known as direction variables) represent decision variables whether circuit $k$ has passed through both neighbor cells $p$ and $p$ or not. $D(p, p)$ is the Euclidean distance between $p$ and $\dot{p}$ :

$$
\begin{aligned}
& D\left(p, p^{\prime}\right)= \\
& \sqrt{\left.(X(p)-X(p)))^{2}+(Y(p)-Y(p))^{2}+(H(p)-H(p))\right)^{2}} .
\end{aligned}
$$

If a line element connects two neighbor cells, then half of the line element will be in one cell and the other half will be in the other cell, where parameter 0.5 is included in Eq. (1). In [12], the following restrictions are considered to limit the direction variables: 
- Non-terminal points: If $p$ is a non-terminal point of candidate circuit $k$ (i.e., circuit $k$ does not start from or end to point $p$ ), circuit $k$ may or may not cross point $p$. If a circuit does not cross point $p$ then binary variables, $\mu_{k}(p, \dot{p})$, are equal to zero for all $\dot{p} \in N(p)$. Otherwise, circuit $k$ crosses point $p$ and two of its neighbors. Therefore, the value of $\Sigma_{\dot{p} \in N(p)} \mu_{k}(p, p ́)$ equals 0 or 2 ;

- Terminal points: If $p$ is a terminal point of circuit $k$, then $\Sigma_{\dot{p} \in N(p)} \mu_{k}(p, \dot{p})$ equals 1 . Otherwise the value of $\Sigma_{\hat{p} \in N(p)} \mu_{k}(p, \hat{p})$ is equal to 0 ;

- Decision variables $\mu_{k}(p, \dot{p})$ and $\mu_{k}(\dot{p}, p)$ are equal for each two-neighbor cells;

- Power lines, routes must be without any loop;

- A power line route must not pass through avoidance areas. Avoidance areas are prohibited to construct transmission lines such as farms, ranges, forests, wetlands, wild life lodging, military zones, etc.;

- If candidate circuit $k$ is not constructed, then all $\mu_{k}(p, p)$ variables are zero.

In this formulation, the possible route of circuit $k$ can be represented as a set of $\mu_{k}$ variables, satisfying the aforementioned restrictions. If $\Lambda_{k}=\left\{\mu_{k}(p, p): p \in\right.$ $M, \dot{p} \in N(p)\}$ is a possible route for circuit $k$, then:

$$
\Sigma_{p \in M} \Sigma_{\hat{p} \in N(p)} 0.5 \mu_{k}(p, \dot{p}) D(p, \dot{p}) B_{k}(p)=y_{k} \times c_{l}\left(\left\{\mu_{k}\right\}\right),
$$

where $y_{k}$ is equal to 1 if circuit $k$ is constructed, and 0 otherwise.

\subsection{SNP reformulation}

The left side of Eq. (4) is equal to construction costs of circuit $k$ if circuit $k$ is decided to be constructed and otherwise it is equal to 0 . Therefore, the objective function of SNP in Eq. (1) is rewritten as:

$$
v=\min \Sigma_{k=1}^{N_{c}} y_{k} \times c_{k}\left(\Lambda_{k}\right) .
$$

Defining $n_{i j}$ as the number of circuits to be constructed in the right-of-way $i-j$, Eq. (5) is rewritten as Eq. (6). All the candidate circuits in the same right-of-way are assumed to have similar properties:

$$
v=\min \Sigma_{(i, j) \in \Omega} n_{i j} c_{i j}\left(\Lambda_{i j}\right) .
$$

The constraints of the proposed formulation are as follows:

Nodal power balance. The following equality constraint represents the conservation of active power at each node:

$$
P_{g}(i)-P_{d}(i)=\Sigma_{(i, j) \in \Omega_{i}} f_{i j} .
$$

Transmission line power flow limit. This inequality constraint is applied to limit the power flow of each transmission line:

$$
\left|f_{i j}\right| \leq\left(n_{i j}^{0}+n_{i j}\right) \bar{f}_{i j} .
$$

This work employs a DCPF model; therefore, power flow through line $i-j$ is calculated using Eq. (9).

$$
f_{i j}=\frac{\left(n_{i j}^{0}+n_{i j}\right)}{x_{i j}} \times\left(\theta_{i}-\theta_{j}\right) .
$$

Due to the impacts of line route on its reactance, Eq. (9) is written as follows:

$$
f_{i j}=\frac{\left(n_{i j}^{0}+n_{i j}\right)}{x_{i j}\left(\Lambda_{i j}\right)} \times\left(\theta_{i}-\theta_{j}\right) .
$$

The right-of-ways with the already existing power circuits are assumed with a pre-defined route; so the value of $x_{i j}$ for the existing lines is kept constant.

Right-of-way limitation. The number of circuits that can be installed in each right-of-way is limited by:

$$
0 \leq n_{i j} \leq \bar{n}_{i j}
$$

Interconnectivity. Here, the interconnectivity of a network is considered.

Based on the aforementioned equations, SNP problem can be written as follows:

$$
v=\min \Sigma_{(i, j) \in \Omega} n_{i j} \times c_{i j}\left(\Lambda_{i j}\right),
$$

subject to:

$$
\begin{aligned}
& \left|f_{i j}\right| \leq\left(n_{i j}^{0}+n_{i j}\right) \bar{f}_{i j}, \\
& f_{i j}=\frac{\left(n_{i j}^{0}+n_{i j}\right)}{x_{i j}\left(\Lambda_{i j}\right)} \times\left(\theta_{i}-\theta_{j}\right), \\
& P_{g}(i)-P_{d}(i)=\Sigma_{(i, j) \in \Omega_{i}} f_{i j}, \\
& 0 \leq n_{i j} \leq \bar{n}_{i j},
\end{aligned}
$$

Interconnectivity.

Considering $\Lambda_{i j}^{r}, r=1, \ldots, R_{i j}$ as different candidate routes in the right-of-way $i-j, \Pi_{i j}$ can be written as:

$$
\begin{aligned}
& \Pi_{i j}:=\left\{(x, c): x=x_{i j}\left(\Lambda_{i j}^{r}\right), c=c_{i j}\left(\Lambda_{i j}^{r}\right),\right. \\
& \left.r=1, \ldots, R_{i j}\right\} .
\end{aligned}
$$

Based on Eq. (13), SNP mathematical framework is presented as:

$$
v=\min \Sigma_{(i, j) \in \Omega} n_{i j} c_{i j},
$$


subject to:

$$
\begin{aligned}
& \left|f_{i j}\right| \leq\left(n_{i j}^{0}+n_{i j}\right) \bar{f}_{i j}, \\
& f_{i j}=\frac{\left(n_{i j}^{0}+n_{i j}\right)}{x_{i j}} \times\left(\theta_{i}-\theta_{j}\right), \\
& \left(x_{i j}, c_{i j}\right) \in \Pi_{i j}, \\
& P_{g}(i)-P_{d}(i)=\Sigma_{(i, j) \in \Omega_{i}} f_{i j}, \\
& 0 \leq n_{i j} \leq \bar{n}_{i j},
\end{aligned}
$$

\section{Interconnectivity.}

It is noted that $\Pi_{i j}$ sets are obtained from the following pre-processing stage.

\section{The line routing preprocessing stage}

In this section, for the sake of simplicity, three following definitions are provided.

Definition 1. A "unit transmission line" is defined as a fictitious type of transmission line having unit construction costs in a typical environment.

Definition 2. A "least cost route" between points $p_{1}$ and $p_{2}$ is the cheapest route between these two points. Considering the least cost route between these two points, the length and construction costs of a unit transmission line are denoted by $l^{L C}\left(p_{1}, p_{2}\right)$ and $c^{u}\left(p_{1}, p_{2}\right)$, respectively.

Suppose that the same type line is selected for right-of-way $i-j$ with the least cost route between two points $p_{1}$ and $p_{2}$. Eqs. (15) and (16) define the construction cost and reactance of each circuit of such a line:

$$
\begin{aligned}
& c_{i j}^{L C}\left(p_{1}, p_{2}\right)=\bar{c}_{i j} \times c^{u}\left(p_{1}, p_{2}\right), \\
& x_{i j}^{L C}\left(p_{1}, p_{2}\right)=\bar{x}_{i j} \times l^{L C}\left(p_{1}, p_{2}\right) .
\end{aligned}
$$

Definition 3. "A route with one degree of obligation" between points $p_{1}$ and $p_{2}$ with obligation point $p$ is the cheapest route to construct a power line between two points $p_{1}$ and $p_{2}$ while it has to pass through point $\not$. Such a route is composed of two parts: a least cost route between points $p_{1}$ and $\dot{p}$ and a least cost route between points $\dot{p}$ and $p_{2}$. In this work, the candidate routes are selected among this kind of routes. Figure 1 shows two sample routes between points $p_{1}$ and $p_{2}$ with different obligation points ( $\mathrm{A}$ and $\mathrm{B}$ ). For example, the route with obligation point $\mathrm{A}$ is composed of two parts: one between $p_{1}$ and $\mathrm{A}$ and the other between $\mathrm{A}$ and $p_{2}$. This definition helps to have a set of candidate routes

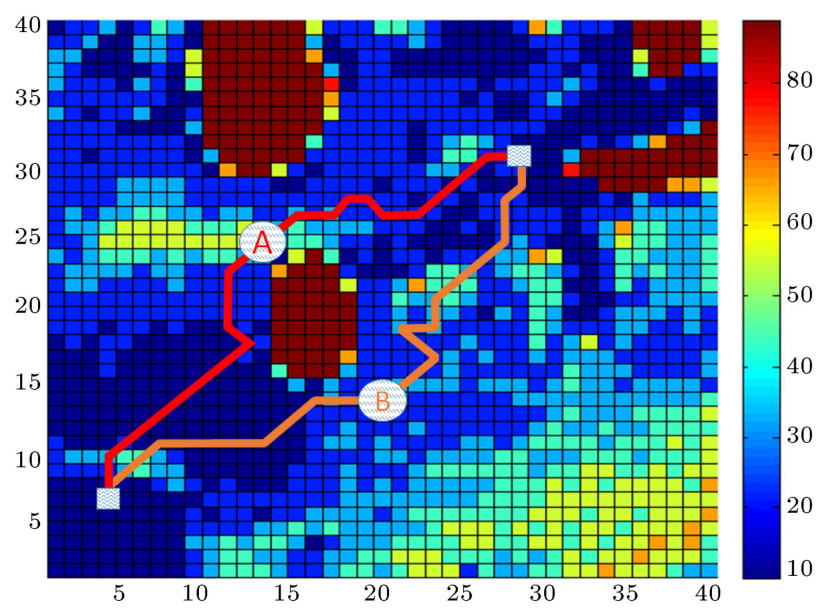

Figure 1. Sample routes with different obligation points $\mathrm{A}$ and $\mathrm{B}$.

whose their lengths as well as construction costs can be calculated easily in the pre-processing stage.

Considering $\dot{p}$ as an obligation point, the reactance and construction costs of a circuit in right-of-way $i-j$ are calculated by Eqs. (18) and (20), respectively:

$$
\begin{aligned}
& c_{i j}(\dot{p})=c_{i j}^{L C}\left(p_{i}^{\text {bus }}, p^{\prime}\right)+c_{i j}^{L C}\left(p_{j}^{\text {bus }}, \dot{p}\right), \\
& c_{i j}\left(p^{\prime}\right)=\bar{c}_{i j} \times\left(c^{u}\left(p_{i}^{\text {bus }}, p^{\prime}\right)+c^{u}\left(p_{j}^{b u s}, p^{\text {us }}\right)\right), \\
& x_{i j}\left(\dot{p}^{\prime}\right)=x_{i j}^{L C}\left(p_{i}^{\text {bus }}, p^{\prime}\right)+x_{i j}^{L C}\left(p_{j}^{\text {bus }}, p^{\prime}\right), \\
& x_{i j}\left(\dot{p}^{\prime}\right)=\bar{x}_{i j} \times\left(l^{L C}\left(p_{i}^{\text {bus }}, \dot{p}^{\prime}\right)+l^{L C}\left(p_{j}^{\text {bus }}, \dot{p}^{\prime}\right)\right) .
\end{aligned}
$$

Definition 4. The reactance class of a route with obligation point $p$ for right-of-way $i-\mathrm{j}$ is defined as:

$$
\left\lfloor\frac{x_{i j}\left(p^{\prime}\right)}{x_{i j}^{L C}\left(p_{i}^{b u s}, p_{j}^{b u s}\right) \times m}\right\rfloor,
$$

where $\lfloor\cdot\rfloor$ denotes a floor function. The proper value of $m$ as a constant can be advised empirically $(0<$ $m<1)$. Between various routes of a specified right-ofway with the same reactance class, the cheapest one is preferred. This classification helps to remove candidate routes with close values of reactances. A too small value of $m$ may cause a large number of candidate routes. Some of those routes might not be acceptable due to cheaper routes with almost the same reactance. In contrast, a very large value of $m$ will result in losing some acceptable candidate routes. To obtain $\Pi_{i j}$ sets, the reactance and construction costs of line $i-j$ for various possible routes should be calculated and stored using the following steps:

Step 1. Calculate $c^{u}\left(p_{i}^{\text {bus }}, p\right)$ and $\left.l^{L C}\left(p_{i}^{\text {bus }}, p\right)\right)$ for each bus $i$ and each point $p \in M$ based on well-known Dijkstra's algorithm [23,24]. 
Step 2. For each $(i, j) \in \Omega^{C}$, only one route denoted by $\Lambda_{i j}$ can be considered as an option:

$$
\Pi_{i j}=\left\{\left(x_{i j}\left(\Lambda_{i j}\right), c_{i j}\left(\Lambda_{i j}\right)\right\}, \forall(i, j) \in \Omega^{C} .\right.
$$

Step 3. For each $(i, j) \in \Omega^{C}$, do Steps 4 to 6 .

Step 4. For each point $\not \dot{p} \in M$, do Steps 5 and 6 .

Step 5. Consider $\not \dot{p}$ as the obligation point of $\Lambda_{i j}$. Calculate $x_{i j}(\dot{p})$ and $c_{i j}(\not ́)$.

Step 6. Add $\left(x_{i j}(\not \dot{p}), c_{i j}(\not ́)\right)$ to set $\Pi_{i j}$. If there is another $(x, c) \in \Pi_{i j}$ in the same reactance class, then keep the cheaper one and remove the more expensive alternative.

\section{The proposed algorithm to solve SNP problem}

Any meta-heuristic method can be modified to solve the proposed model, such as those presented in $[3,25]$. The key aspects of implementing a meta-heuristic technique are coding and evaluating solutions. The coding refers to representing a candidate solution in the search space. Moreover, the algorithm defines a scheme to handle infeasible solutions and calculate the objective function. The encoding is similar to the ones employed by most genetic algorithm implementations [26-28]. The major difference is the meaning of each element in the coding vector. Elements of the coding vector indicate the candidate route selected for each right-ofway as well as the number of transmission lines added in the route.

In the beginning, the algorithm performs the pre-processing stage described in Section 3 for once. Having the pre-processed data (i.e., $\Pi_{i j}$ ), the objective function can be calculated for each candidate solution. To evaluate a candidate solution, first, simply read the values of $\left(x_{i j}, c_{i j}\right) \in \Pi_{i j}$ according to the selected candidate path for each right-of-way. In order to check the feasibility of a solution, a DCPF should perform and check the line overflows Relation (14a) as well as the number of added line limits Relation (14e) and network interconnectivity Relation (14f). Most of the meta-heuristic techniques can be adopted to use such coding and evaluation scheme. In the present case, minor changes were applied to the algorithm presented in [29] to solve the proposed expansion problem as it is illustrated in Figure 2.

\section{Case studies}

In this section, the proposed methodology is applied to four case studies. The first test case is a 3-bus test system on which the concept of the proposed methodology is explained via a numerical example. The second case is the 18-bus test system to compare the performance and effectiveness of the proposed methodology with those of [12]. In addition, the original and modified versions of 51-bus southeast network of Iran are studied as practical systems.

\subsection{3-bus test system}

This case study is performed to explain the proposed methodology via a numerical study. Figure 3 shows the topology of the considered 3-bus system. System data are provided in Tables 1 and 2 .

The GIS data of this system are presented by a $15 \times 15$ rasterized map where each cell represents a $10 \mathrm{~km} \times 10 \mathrm{~km}$ area. Figure 4 shows the cost map of this system, where blue cells represent typical environment and cost factor in pink-colored areas is 3 . The altitudes of all cells are assumed identical. Employing Dijkstra's algorithm, the values of $c^{u}\left(p_{i}^{\text {bus }}, p\right)$ and $l^{L C}\left(p_{i}^{\text {bus }}, p\right)$ are obtained as in Figure 5. Using Relations (8) and (20), the values of $c_{1-2}(p)$ and $x_{1-2}(p)$ are obtained for each point of the map as in Figure 6 . $\left.c_{1-2}(p)\right)$.

Figure 7 illustrates different values of $\left(x_{1-2}(p)\right.$,

Considering the least-cost route for lines 1-2, cost and reactance of any additional circuit would be $27 \mathrm{M} \$$ and 13.5 p.u, respectively. Therefore, using the classical TEP formulation, the optimal solution results in an investment of $54 \mathrm{M} \$$, consisting of adding two

Table 1. Generations and loads of 3-bus test system.

\begin{tabular}{ccccc}
\hline Bus & $\begin{array}{c}\text { Generation } \\
(\mathbf{M W})\end{array}$ & $\begin{array}{c}\text { Load } \\
(\mathbf{M W})\end{array}$ & X coordination & Y coordination \\
\hline 1 & 100 & 0 & 2 & 2 \\
2 & 0 & 100 & 14 & 2 \\
3 & 0 & 0 & N/A & N/A \\
\hline
\end{tabular}

Table 2. Circuit data of 3-bus test system.

\begin{tabular}{cccccccc}
\hline From bus & To bus & $\boldsymbol{n}_{\mathbf{0}}$ & $\boldsymbol{x}_{\boldsymbol{i} \boldsymbol{j}}$ (p.u.) & $\overline{\boldsymbol{x}}_{\boldsymbol{i} \boldsymbol{j}}$ (p.u.) & $\boldsymbol{c}_{\boldsymbol{i} \boldsymbol{j}}(\mathbf{M} \$)$ & $\overline{\boldsymbol{c}}_{\boldsymbol{i} \boldsymbol{j}}(\mathbf{M} \$)$ & $\boldsymbol{f}_{\boldsymbol{i} \boldsymbol{j}}(\mathbf{M W})$ \\
\hline 1 & 2 & 0 & $\mathrm{~N} / \mathrm{A}$ & 0.1 & $\mathrm{~N} / \mathrm{A}$ & 0.2 & 50 \\
1 & 3 & 1 & 10 & $\mathrm{~N} / \mathrm{A}$ & 20 & $\mathrm{~N} / \mathrm{A}$ & 30 \\
2 & 3 & 1 & 10 & $\mathrm{~N} / \mathrm{A}$ & 20 & N/A & 30 \\
\hline
\end{tabular}




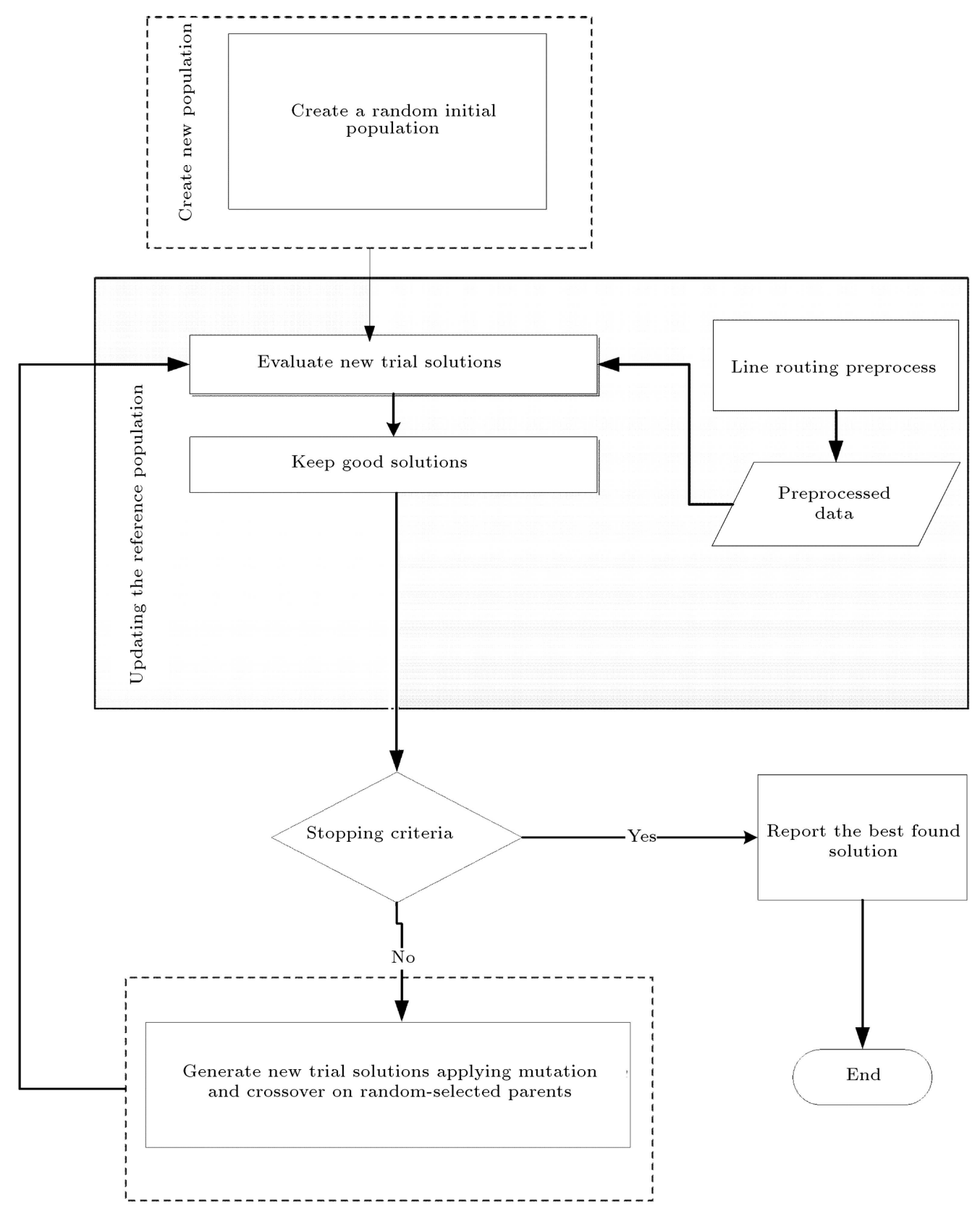

Figure 2. The proposed algorithm to solve SNP problem.

transmission lines in the right-of-way 1-2. Figure 8 shows the optimal topology with the corresponding active power flow distribution.

Now, consider another option for lines 1-2 which $c_{1-2}=32 \mathrm{M} \$$ and $x_{1-2}=12$ p.u. As depicted in Figure 9, in this case, the addition of a single line in right-of-way 1-2 is sufficient and the resulting cost is $32 \mathrm{M} \$$. It can be observed that the least-cost route is not always optimum.

As observed in this case study, the proposed methodology results in a different network topology as compared to the classical model. As discussed in the previous sections, the line-routing problem can influence the optimal transmission network topology.
The proposed model may obtain routes that are more expensive, but it can lower the overall expansion costs. Having understood the differences brought about by considering line routing sub-problem, the next task is to evaluate the effectiveness and performance of the proposed methodology.

\subsection{The 18-bus test system}

In this case study, performance and effectiveness of the proposed methodology are compared with those of [12]. The system includes 18 busses, 28 right-of-ways for addition of new circuits with $35870 \mathrm{MW}$ demand for the base topology. The GIS data of this system are presented by a $40 \times 40$ rasterized map. The system data 


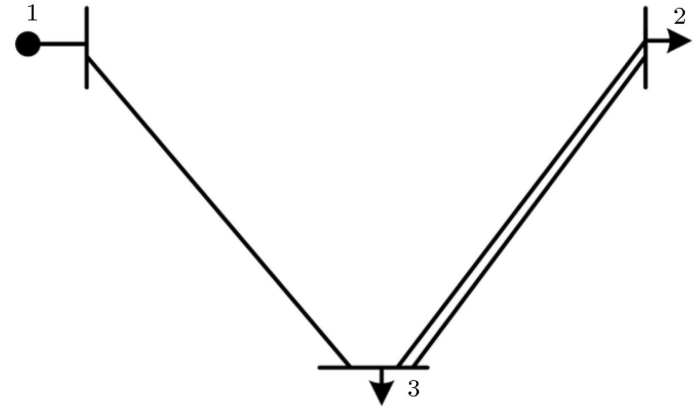

Figure 3. Single diagram of the three-bus system.

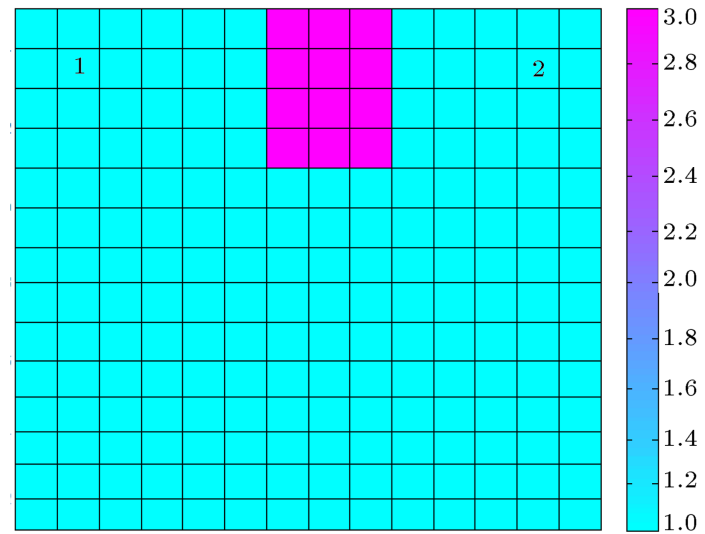

Figure 4. Cost map of the 3-bus system.

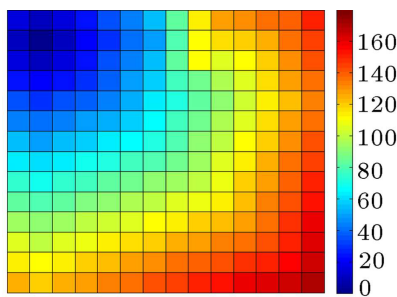

(a) $c^{u}\left(p_{1}^{\text {bus }}, p\right)$

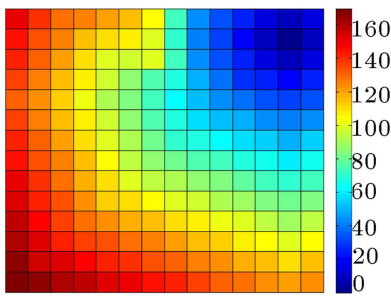

(c) $c^{u}\left(p_{2}^{\text {bus }}, p\right)$

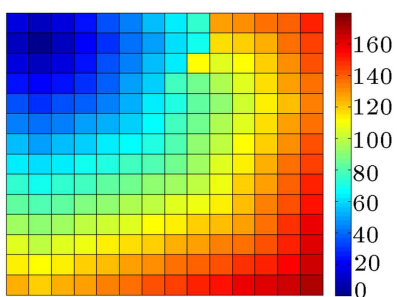

(b) $l^{L C}\left(p_{1}^{\text {bus }}, p\right)$

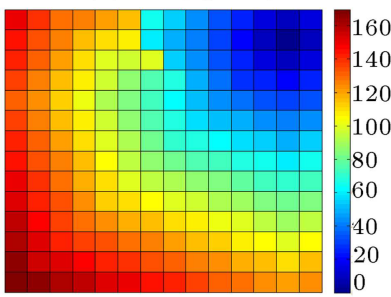

(d) $l^{L C}\left(p_{2}^{\text {bus }}, p\right)$
Figure 5. The results of Dijkstra's algorithm.

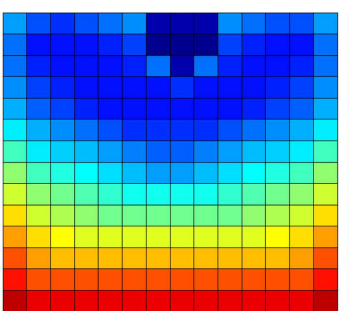

(a) $x_{1-2}(p)$
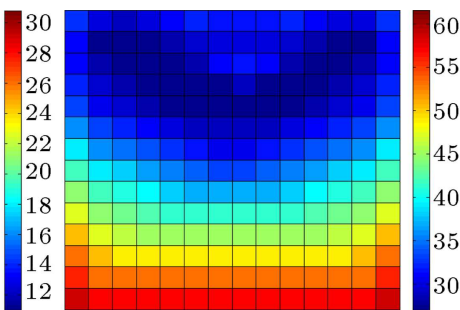

(b) $c_{1-2}(p)$
Figure 6. Cost and reactance of a circuit in line 1-2 as functions of obligation point. can be found in $[12,30]$. Table 3 compares the obtained results with those of [12]. It can be seen through numerical results that the proposed algorithm finds a more economical solution with less computational effort.

As illustrated in Table 4 and Figure 10, the route of line 4-16 is modified to help diminish power flow violations. Figure 11 illustrates the relation between the desired reactance and the construction cost of this line. This figure shows that a significant change in the line reactance can be obtained at rather little extra cost. Similar to the 3-bus system, the planner can choose between short routes through high-cost lands and long routes through low-cost areas. Despite the 3 -bus system, in this case, the optimization problem tends to choose a longer route for lines 4-6 to increase the reactance of this line. As it can be observed in this

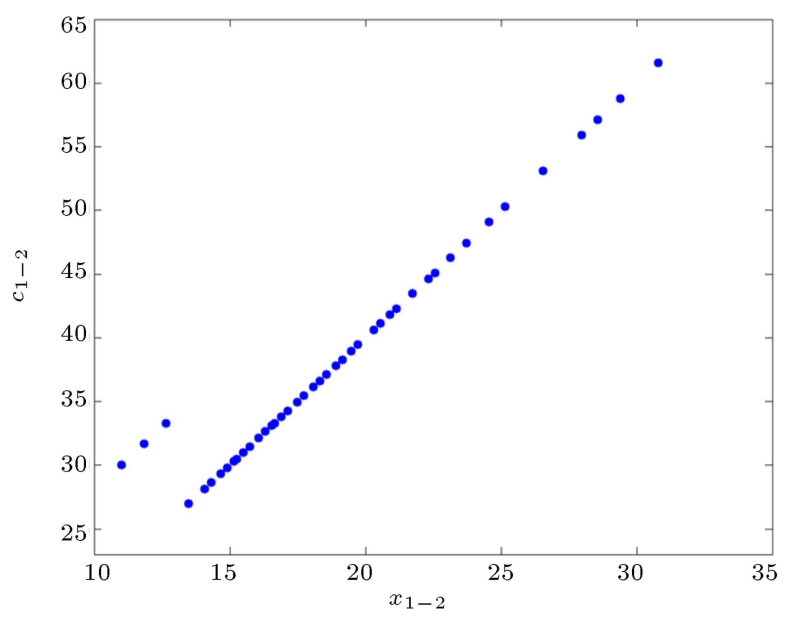

Figure 7. The obtained relation between construction cost and desired reactance of line 1-2.

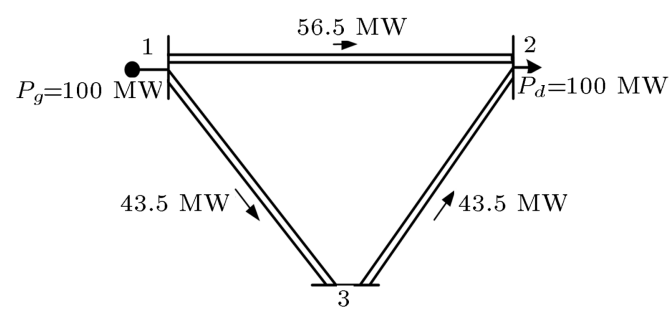

Figure 8. Optimal topology obtained with classical methodology.

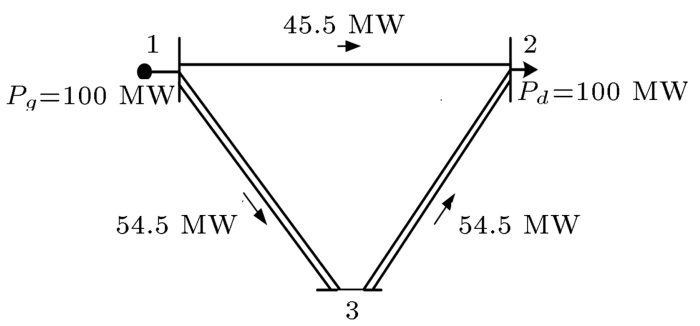

Figure 9. Optimal topology obtained with the proposed methodology. 
Table 3. The obtained optimal solution for 18-bus system.

\begin{tabular}{ccc}
\hline Method & Added circuits & Total investment cost $\left(\mathbf{1 0}^{\mathbf{3}} \$\right)$ CPU time (s) \\
\hline & $n_{1,11}=1, n_{4,16}=1, n_{5,12}=1$, & \\
June et al. [12] & $n_{6,14}=1, n_{7,15}=1, n_{10,18}=1$, & 3887.80 \\
& $n_{11,13}=1, n_{12,13}=1, n_{14,15}=1$, & \\
& $n_{16,17}=1, n_{17,18}=1$. \\
& \\
& $n_{1,11}=1, n_{4,7}=1, n_{4,16}=2$, \\
The proposed algorithm & $n_{5,12}=1, n_{6,14}=1, n_{10,18}=1$, & \\
& $n_{12,13}=1, n_{14,15}=1, n_{17,18}=1$. & \\
\hline
\end{tabular}

Table 4. The optimal routes for candidate lines.

\begin{tabular}{cccc}
\hline Line & Route & $\frac{\boldsymbol{x}_{\boldsymbol{i j}}}{\boldsymbol{x}_{\boldsymbol{i}}^{L C}\left(p_{\boldsymbol{i}}^{\text {bus }}, \boldsymbol{p}_{j}^{\text {bus }}\right)}$ & $\frac{\boldsymbol{c}_{\boldsymbol{i j}}}{\boldsymbol{c}_{\boldsymbol{i}}^{L C}\left(p_{\boldsymbol{i}}^{\text {bus }}, p_{j}^{\text {bus }}\right)}$ \\
\hline $1-11$ & The least cost route & 1 & 1 \\
$4-7$ & The least cost route & 1 & 1 \\
$4-16$ & With obligation point $(36,19)$ & 1.779 & 1.168 \\
$5-12$ & The least cost route & 1 & 1 \\
$6-14$ & The least cost route & 1 & 1 \\
$10-18$ & The least cost route & 1 & 1 \\
$12-13$ & The least cost route & 1 & 1 \\
$14-15$ & The least cost route & 1 & 1 \\
$17-18$ & The least cost route & 1 & 1 \\
\hline
\end{tabular}

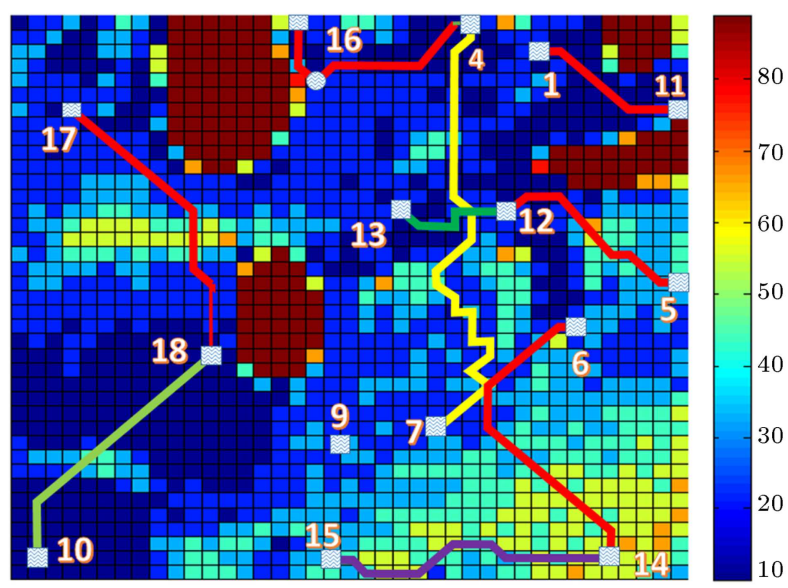

(a)

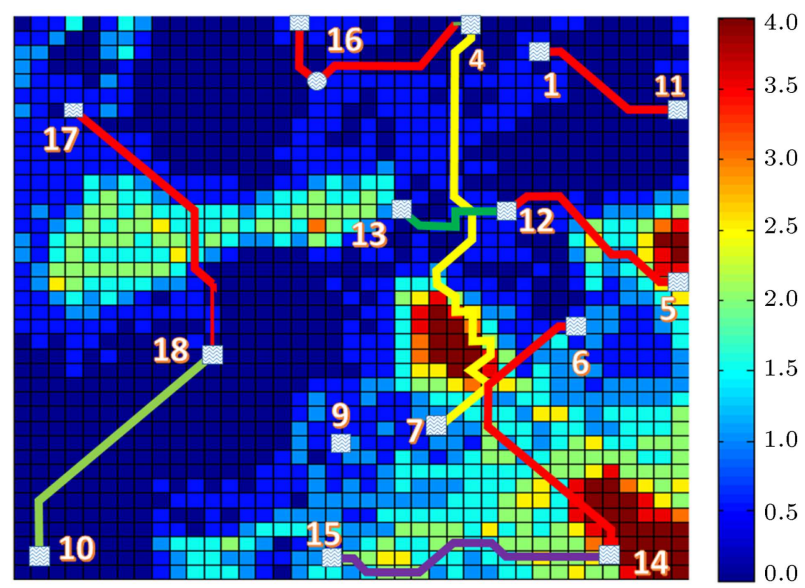

(b)

Figure 10. The optimal result for 18-bus test system: (a) Accompanied with cost map, and (b) accompanied with altitude map.

case study, choosing the least cost routes is expected for most of the power lines. However, modification of power line routes is a useful option in some cases.

\subsection{1 buses belonging to southeast network of Iran}

In this section, the proposed method is applied to the southeast network of Iran. This case study is performed to demonstrate the ability of the proposed algorithm to handle practical power systems with high- resolution GIS rasterized maps. This system has 51 buses, 91 right-of-ways, and a total demand of $10268 \mathrm{MW}$. The base year topology is 2010 and the expansion is targeted for the year 2016. Single-line diagram of southeast network of Iran is illustrated in Figure 12. The GIS data of this system are presented by a $1067 \times 1344$ rasterized map. The presented rasterized map covers a $1000.75 \mathrm{~km} \times 1245.38 \mathrm{~km}$ area. Data of this system are provided in [31]. By applying the proposed algorithm to southeast network of Iran, 
the optimal solution is obtained as in Table 5 and Figure 13.

All optimal routes consist of the least cost routes, meaning that, in this case, the optimal solution of

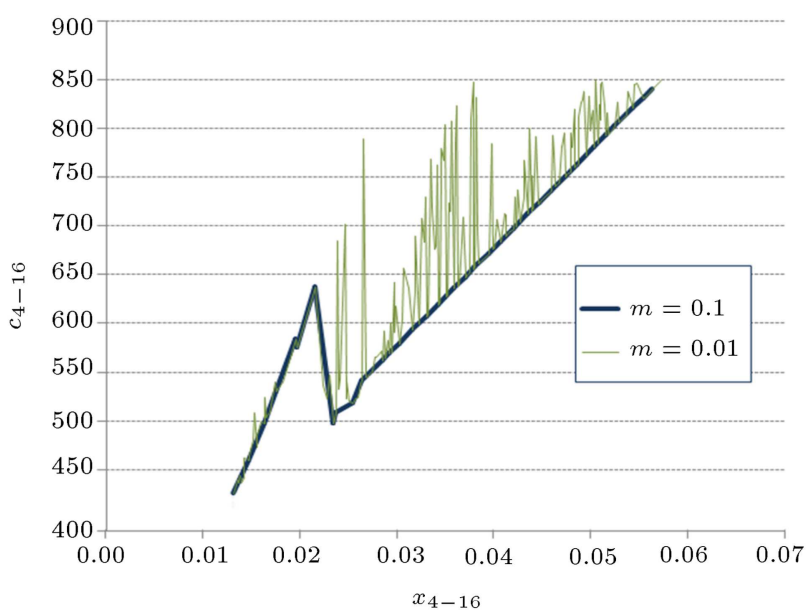

Figure 11. The construction cost of line 4-16 as a function of its desired reactance in 18-bus test system.
SNP is the same as that of traditional TEP problem. The reason is that the environment in this case is not complicated enough. Such non-complexity can be implied by the simple relations between construction costs and desired values for reactance of power lines (see Figure 14 as an example). In such a case, a considerable modification in a power line reactance entails a rather large extra cost.

\subsection{Modified version of southeast network of Iran}

As depicted in Figure 15, the southeast network of Iran is modified in this case study. The applied modifications are: adding $300 \mathrm{MWs}$ of load on bus 17 and 300 MWs of generation on bus 7, adding two constructed lines in the right-of-ways 4-17 and 6-17 in the base topology, and to candidate the right-of-way 7-17. The modified data are provided in [31]. By applying these modifications, the optimal solution is obtained as in Table 6 .

As illustrated in Table 7 and Figure 16, the obligation point of $(879,930)$ is chosen for routes of

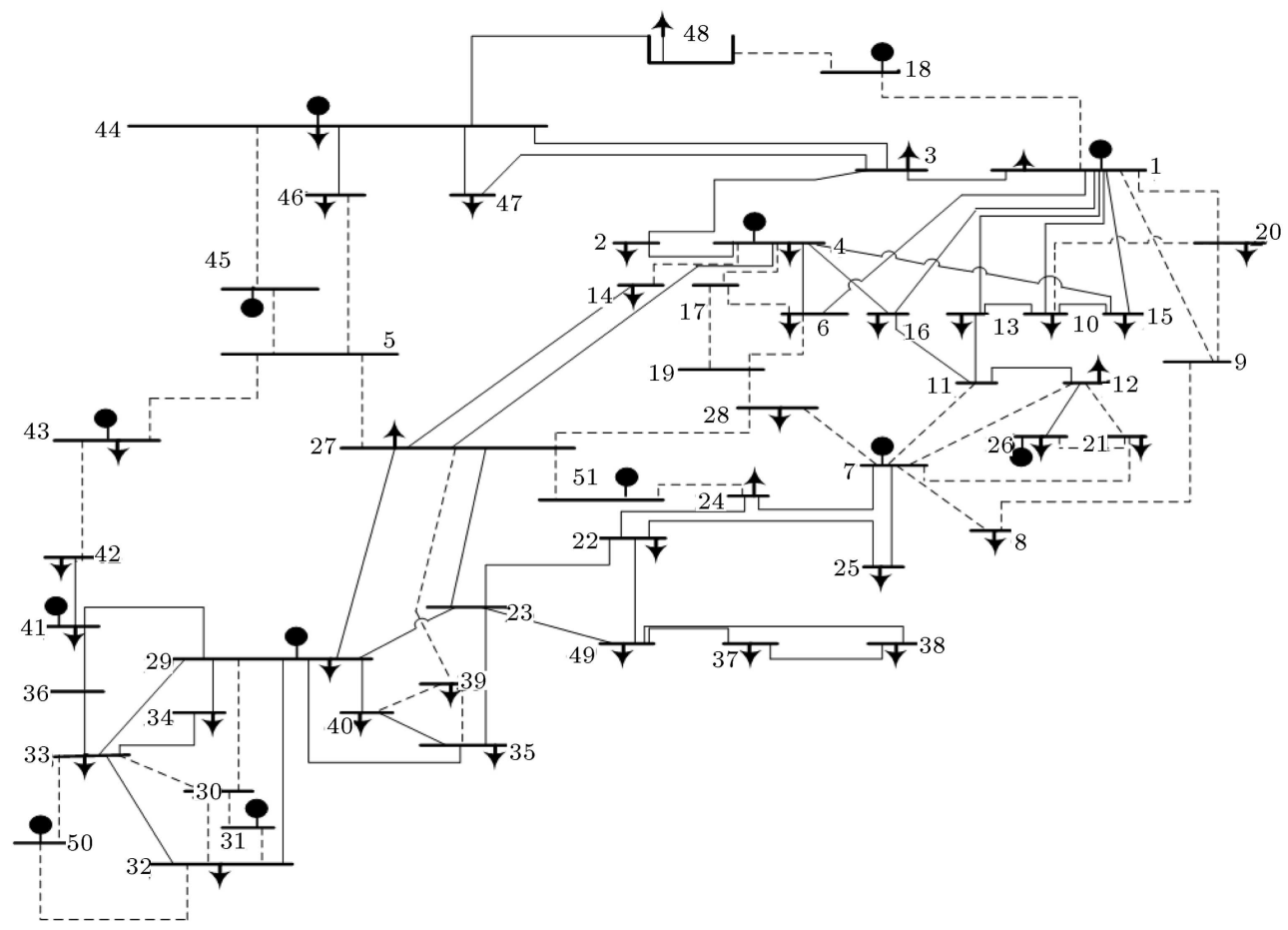

Figure 12. Single diagram of Iran southeast Network.

Table 5. The obtained optimal solution for Iran southeast network.

\begin{tabular}{ccc}
\hline Added circuits & $\begin{array}{c}\text { Total investment } \\
\text { cost }\left(\mathbf{1 0}^{\mathbf{3}} \mathbf{\$}\right)\end{array}$ & $\begin{array}{c}\text { CPU time } \\
\mathbf{( s )}\end{array}$ \\
\hline$n_{7,21}=1, n_{7,28}=1, n_{21,26}=1$, & & \\
$n_{1,18}=1, n_{7,8}=1, n_{10,20}=1$, & 6547.52 & 49707 \\
$n_{35,39}=1, n_{24,51}=2, n_{33,50}=1$, & & \\
$n_{42,43}=1, n_{31,32}=1, n_{44,45}=1$. & & \\
\hline
\end{tabular}




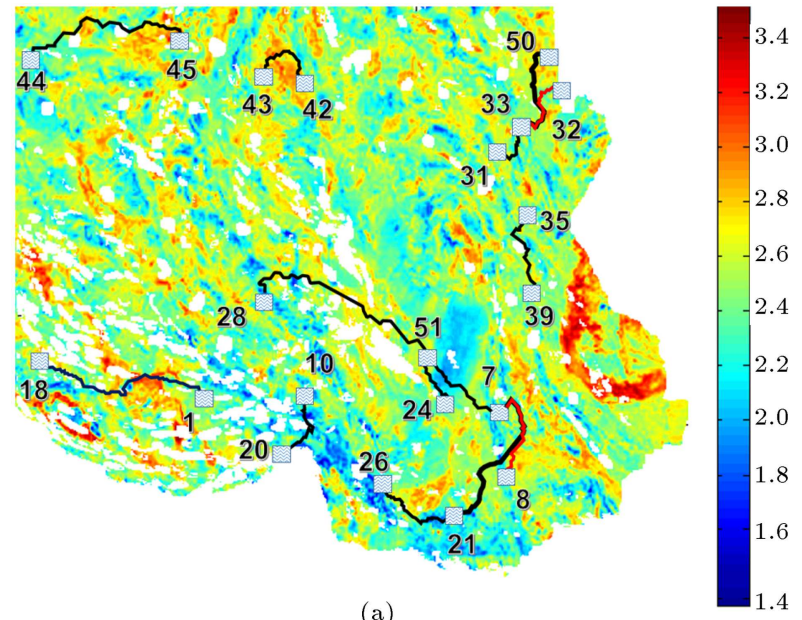

(a)

Figure 13. The optimal result for 51 -bus test system: map. White colour indicates avoidance areas.

lines 7-17. This results in a less-expensive solution in comparison with classical planning. In this case study, constructing a line in right-of-way $7-17$ is a relatively less expensive option to satisfy operational constraints.

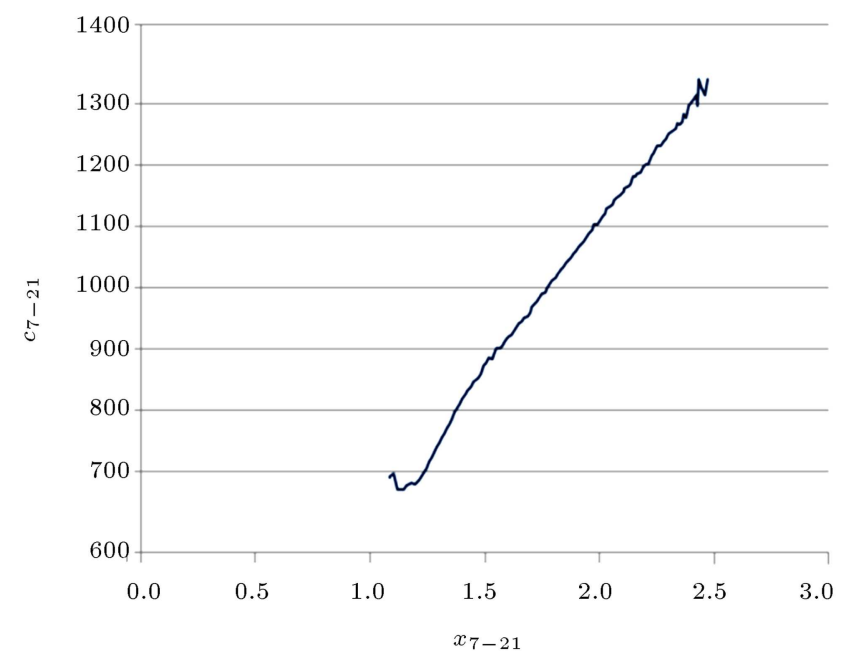

Figure 14. The construction cost of line $7-21$ as a function of its desired reactance in southeast Network.

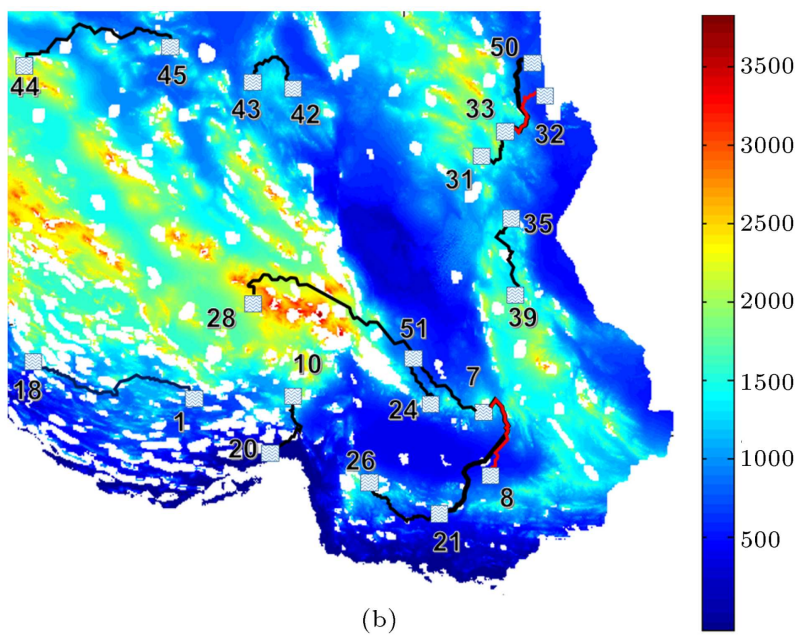

(a) Accompanied with cost map and (b) accompanied with altitude

Therefore, the algorithm decreases its reactance to pass more power flow through this line.

In fact, this test system is intentionally modified to show the effectiveness of the algorithm. In order to investigate this effectiveness, it is necessary to perform various studies on real systems. However, it can be seen that in some cases, modification of line routes is a useful option to obtain more-optimum solutions.

\section{Conclusion}

This paper elaborates on a methodology to solve Spatial Network Planning (SNP). The main modifications and innovations of this work are as follows:

- Providing a new formulation for SNP problem, where the proposed formulation makes it possible to handle line routing sub-problem using pre-processed data;

- Providing pre-processed data based on optimal line routing. The proposed methodology employs a preprocessing stage to reduce the computational effort of line routing sub-problem;

Table 6. The obtained optimal solution for the modified version of Iran southeast network.

\begin{tabular}{|c|c|c|}
\hline Method & Added circuits & Total investment cost $\left(10^{3} \$\right)$ \\
\hline The proposed methodology & $\begin{array}{l}n_{7,21}=1, n_{7,28}=1, n_{21,26}=1, \\
n_{1,18}=1, n_{7,8}=1, n_{10,20}=1, \\
n_{7,17}=2, n_{35,39}=1, n_{24,51}=2, \\
n_{33,50}=1, n_{42,43}=1, n_{31,32}=1, \\
n_{44,45}=1\end{array}$ & 6762.34 \\
\hline Classical planning & $\begin{array}{l}n_{7,21}=1, n_{7,28}=1, n_{21,26}=1, \\
n_{1,18}=1, n_{7,8}=1, n_{10,20}=1, \\
n_{7,13}=2, n_{35,39}=1, n_{24,51}=2, \\
n_{33,50}=1, n_{42,43}=1, n_{31,32}=1, \\
n_{44,45}=1 .\end{array}$ & 6977.16 \\
\hline
\end{tabular}




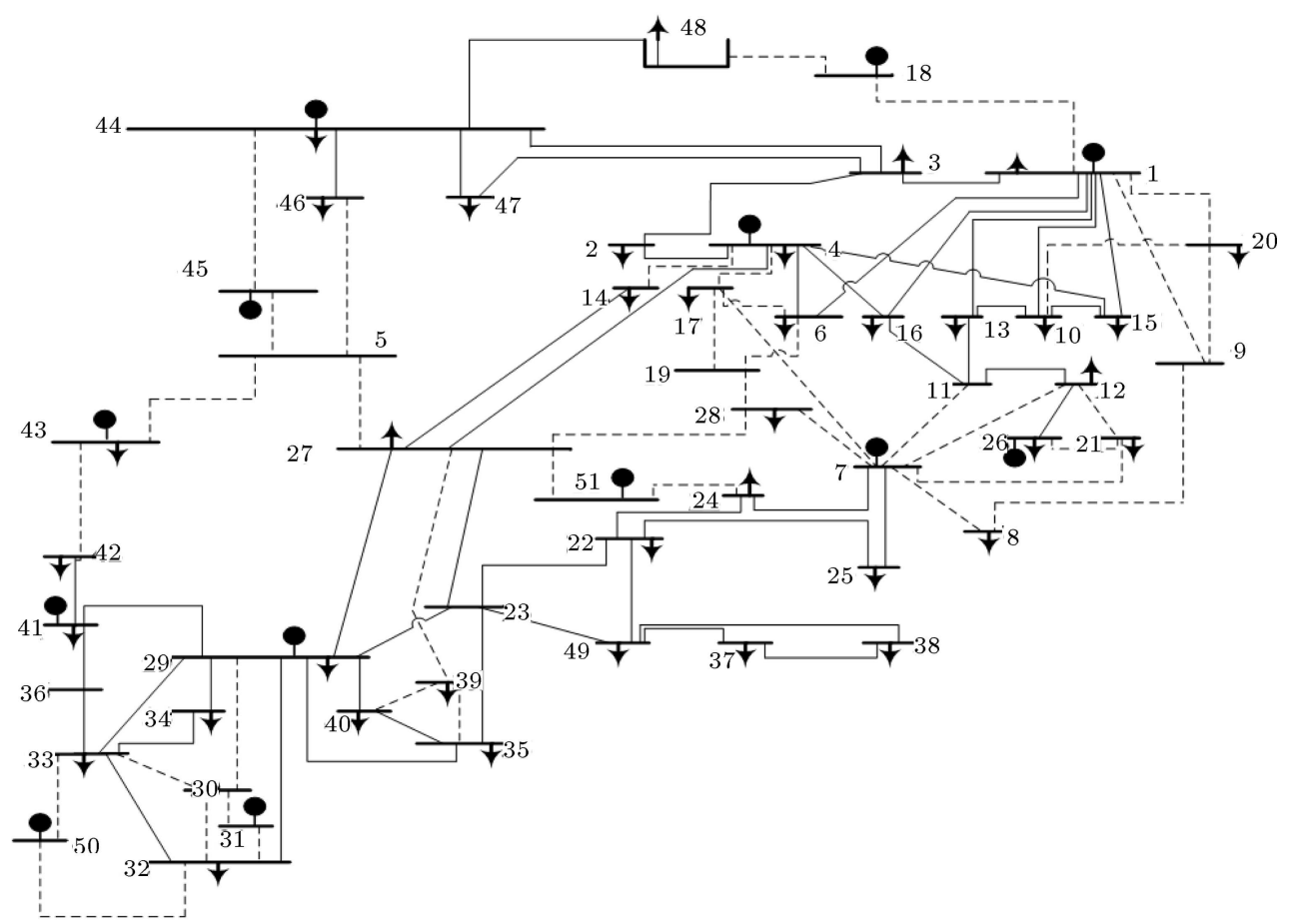

Figure 15. Single diagram of the modified version of Iran southeast network.

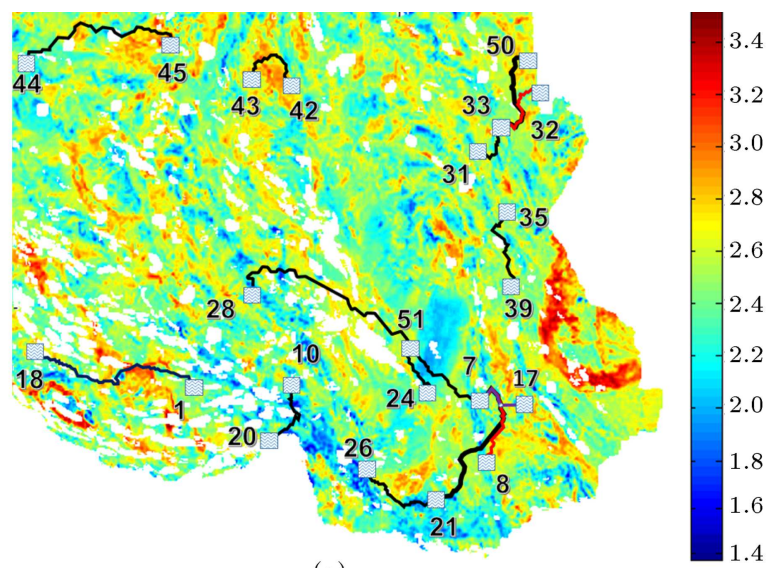

(a)

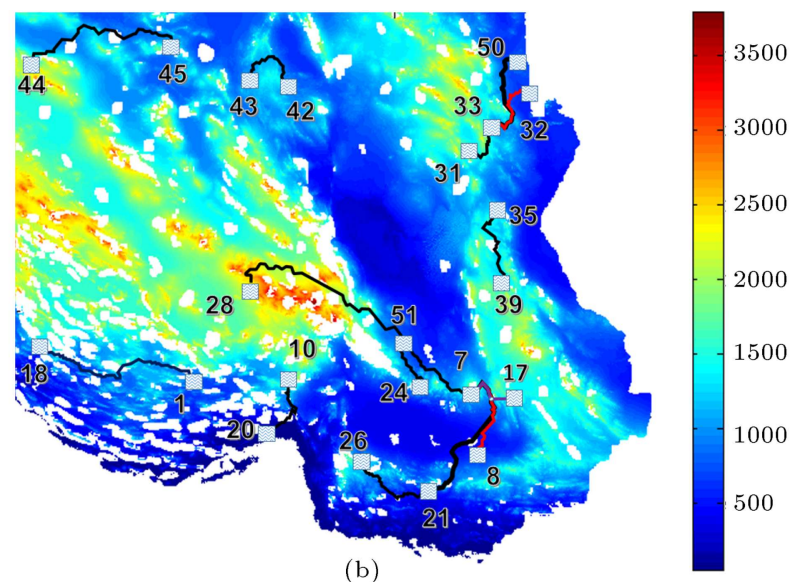

(b)

Figure 16. The optimal result for the modified version of 51-bus test system: (a) Accompanied with cost map and (b) accompanied with altitude map. White colour indicates avoidance areas.

Table 7. The optimal routes for candidate lines.

\begin{tabular}{cccc}
\hline Line & Route & $\frac{\boldsymbol{x}_{i j}}{\boldsymbol{x}_{i j}^{L C}\left(p_{i}^{\text {bus }}, p_{j}^{\text {bus }}\right)}$ & $\frac{\boldsymbol{c}_{i j}}{\boldsymbol{c}_{i j}^{L C}\left(p_{i}^{\text {bus }}, p_{j}^{\text {bus }}\right)}$ \\
\hline $7-21$ & The least cost route & 1 & 1 \\
$7-28$ & The least cost route & 1 & 1 \\
$21-26$ & The least cost route & 1 & 1 \\
$1-18$ & The least cost route & 1 & 1 \\
$7-8$ & The least cost route & 1 & 1 \\
$10-20$ & The least cost route & 1 & 1 \\
$7-17$ & With obligation point (879,930) & 0.783 & 1.43 \\
$35-39$ & The least cost route & 1 & 1 \\
$24-51$ & The least cost route & 1 & 1 \\
$33-50$ & The least cost route & 1 & 1 \\
$42-43$ & The least cost route & 1 & 1 \\
$31-32$ & The least cost route & 1 & 1 \\
$44-45$ & The least cost route & 1 & 1 \\
\hline
\end{tabular}


- Employing a meta-heuristic procedure to solve such a problem is studied. The proposed methodology avoids using classical optimization methods (e.g. linear programming) to solve the SNP problem. Here a modification in the algorithm is easier, and this can simplify the future research studies in this area.

The results obtained from test systems illustrate the effectiveness of the proposed framework. It is observed through numerical studies that the proposed algorithm is able to solve the SNP problem even for large and complex systems with high-resolution GIS rasterized maps. In the following, limitations of the proposed methodology and ideas to improve this work are discussed:

- In this work, a route is considered as an integration of vertical, horizontal, and diagonal elements. Therefore, the length of a route can be overestimated due to triangular inequality. More advanced line routing methods can improve the accuracy of the model. Considering a larger neighborhood can provide elements in various directions to enhance the effectiveness of the model;

- Locating new substations should be considered in TEP problem. Therefore integration of SNP with substation expansion planning problem is suggested for future studies;

- The proposed algorithm solves a single-stage DC problem that neglects various aspects of TEP problem including reactive power flows, voltage variations, stability, etc. Therefore, modification of the proposed method to solve an extended SNP problem is suggested, considering multi-objective planning models, uncertain models, multistage planning, etc.;

- In most cases, the land costs of each point cannot be easily evaluated. Therefore, new probabilistic models are needed to deal with incomplete and inaccurate GIS data;

- In this work, candidate routes are chosen among those with one degree of obligation. Considering a wider range of routes, which may improve the method, is suggested.

\section{Nomenclature}

\section{Indices}

$j$

$k$

\section{Sets}
$\Omega^{C}$
The set of right-of-ways containing already constructed circuits

\section{$\Omega^{U}$}
$\Omega_{i}$
$\Pi_{i j}$
$M \quad$ The set of all center points of cells in
The set of candidate right-of-ways not belonging to $\Omega^{C}$
$\Omega_{i} \quad$ The set of right-of-ways connected to bus $i$
The set of pre-processed data for right-of-way $i-j$ the rasterized map
$N(p) \quad$ The set of all neighbor points of $p$

\section{Variables}

$\Lambda_{i j} \quad$ The route of line $i-j$

$\Lambda_{i j}^{r} \quad$ The $r$ th candidate route in right-of-way $i-j$

$\mu_{k}\left(p, p^{\prime}\right) \quad$ The binary variable represents the decision whether circuit $k$ has passed through both neighbour cells $p$ and $p^{\prime}$ or not

$\theta_{i} \quad$ Phase angle at bus $i$

$c_{i j}(p) \quad$ The investment cost of one circuit in right-of-way $i-j$ in a route with obligation point $p$

$c_{i j} \quad$ The investment cost of one circuit in right-of-way $i-j$

$c_{i j}^{L C}\left(p_{i}, p_{j}\right) \quad$ The construction cost of a line with the same type considered for right-of-way $i-j$ in the least-cost route between two points $p_{i}$ and $p_{j}$

$D\left(p_{i}, p_{j}\right) \quad$ Euclidean distance between $p_{i}$ and $p_{j}$ $f_{i j} \quad$ Active power flow through line $i-j$

$l^{L C}\left(p_{i}, p_{j}\right) \quad$ Length of the least-cost route between two points $p_{i}$ and $p_{j}$

$n_{i j} \quad$ The number of circuits added to right-of-way $i-j$

$v \quad$ The objective function value of spatial network planning problem

$x_{i j}(p) \quad$ Reactance of one circuit in right -of-way $i-j$ in a route with obligation point $p$

$x_{i j}^{L C}\left(p_{i}, p_{j}\right) \quad$ Reactance of one circuit with the same type considered for right-of- way $i-j$ in the least-cost route between two points $p_{i}$ and $p_{j}$.

$y_{k}$

Decision variable of circuit $k$ construction.

\section{References}

1. Romero, R., Rider, M.J., and Silva, I.D.J. "A metaheuristic to solve the transmission expansion plan- 
ning", Power Systems, IEEE Transactions on, 22, pp. 2289-2291 (2007).

2. Faria, H. Jr., Binato, S., Resende, M.G.C., and Falcao, D.M. "Power transmission network design by greedy randomized adaptive path relinking", Power Systems, IEEE Transactions on, 20, pp. 43-49 (2005).

3. Sum-Im, T., Taylor, G., Irving, M., and Song, Y. "Differential evolution algorithm for static and multistage transmission expansion planning", Generation, Transmission \& Distribution, IET, 3, pp. 365-384 (2009).

4. Hemmati, R., Hooshmand, R.A., and Khodabakhshian, A. "Comprehensive review of generation and transmission expansion planning", Generation, Transmission \& Distribution, IET, 7, pp. 955-964 (2013).

5. Yongfu, L., Qing, Y., Wenxia, S., Jiaqi, L., and Tao, $Y$. "Optimization of transmission-line route based on lightning incidence reported by the lightning location system", Power Delivery, IEEE Transactions on, 28, pp. $1460-1468$ (2013).

6. Monteiro, C., Ramirez-Rosado, I.J., Miranda, V., Zorzano-Santamaria, P.J., Garcia-Garrido, E., and Fernandez-Jimenez, L.A. "GIS spatial analysis applied to electric line routing optimization", Power Delivery, IEEE Transactions on, 20, pp. 934-942 (2005).

7. Vega, M. and Sarmiento, H.G. "Image processing application maps optimal transmission routes", Computer Applications in Power, IEEE, 9, pp. 47-51 (1996).

8. West, N., Dwolatzky, B., and Meyer, A. "Terrain based routing of distribution cables", Computer Applications in Power, IEEE, 10, pp. 42-46 (1997).

9. Luchmaya, A., Dwolatzky, B., and Meyer, A. "Using terrain information in an electrification planning tool", in Transmission and Distribution Conference and Exposition, 2001 IEEE/PES, pp. 456-460 (2001).

10. Sumic, Z., Venkata, S., and Pistorese, T. "Automated underground residential distribution design. I. Conceptual design", Power Delivery, IEEE Transactions on, 8, pp. 637-643 (1993).

11. Sumic, Z., Pistorese, T., Males-Sumic, H., and Venkata, S. "Automated underground residential distribution design. II. Prototype implementation and results", Power Delivery, IEEE Transactions on, 8, pp. 644-650 (1993).

12. Jun, S., Lei, W., Zuyi, L., Shahidehpour, M., Li-Zi, Z., and Bing, H. "A new method for spatial power network planning in complicated environments", Power Systems, IEEE Transactions on, 27, pp. 381-389 (2012).

13. Yeh, E.-C. and Tram, H. "Information integration in computerized distribution system planning", in Transmission and Distribution Conference, Proceedings., IEEE, pp. 602-607 (1996).
14. Correa, C.A., Bolanos, R.A., and Garces, A. "Environmental transmission expansion planning using non-linear programming and evolutionary techniques", in Alternative Energies and Energy Quality (SIFAE), 2012 IEEE International Symposium on, pp. 1-5 (2012).

15. Hui, Z., Vittal, V., Heydt, G.T., and Quintero, J. "A mixed-integer linear programming approach for multi-stage security-constrained transmission expansion planning", Power Systems, IEEE Transactions on, 27, pp. 1125-1133 (2012).

16. Jabr, R.A. "Optimization of AC transmission system planning", Power Systems, IEEE Transactions on, 28, pp. 2779-2787 (2013).

17. Maghouli, P., Hosseini, S.H., Buygi, M.O., and Shahidehpour, M. "A scenario-based multi-objective model for multi-stage transmission expansion planning", Power Systems, IEEE Transactions on, 26, pp. 470-478 (2011).

18. Rahmani, M., Romero, R., and Rider, M.J. "Strategies to reduce the number of variables and the combinatorial search space of the multistage transmission expansion planning problem", Power Systems, IEEE Transactions on, 28, pp. 2164-2173 (2013).

19. Chatthaworn, R. and Chaitusaney, S. "Substation and transmission line expansion planning using Adaptive Tabu search algorithm", in Electrical Engineering/Electronics, Computer, Telecommunications and Information Technology (ECTI-CON), 2013 10th International Conference on, pp. 1-6 (2013).

20. Ergun, H., Rawn, B., Belmans, R., and Van Hertem, D. "Technology and topology optimization for multizonal transmission systems", in IEEE Transactions on Power Systems, 29(5), pp. 2469-2477 (Sept. 2014).

21. Shu, J., Wu, L., Zhang, L., and Han, B. "Spatial power network expansion planning considering generation expansion", Power Systems, IEEE Transactions on, pp. 1-10 (2014).

22. Shu, J., Wu, L., Han, B., and Zhang, L. "Enhanced multidimensional power network planning based on ant colony optimization", International Transactions on Electrical Energy Systems, 25, pp. 1024-1222 (2015).

23. Dijkstra, E.W. "A note on two problems in connexion with graphs", Numerische Mathematik, 1, pp. 269-271 (1959).

24. Jasika, N., Alispahic, N., Elma, A., Ilvana, K., Elma, L., and Nosovic, N. "Dijkstra's shortest path algorithm serial and parallel execution performance analysis", in MIPRO, 2012 Proceedings of the 35th International Convention, pp. 1811-1815 (2012).

25. Verma, A., Panigrahi, B., and Bijwe, P. "Harmony search algorithm for transmission network expansion planning", Generation, Transmission \& Distribution, IET, 4, pp. 663-673 (2010).

26. Escobar, A., Gallego, R., and Romero, R. "Using traditional heuristic algorithms on an initial genetic 
algorithm population applied to the transmission expansion planning problem", Revista Ingeniera e Investigacin Universidad Nacional de Colombia, 31, pp. 127-143 (2011).

27. Gallego, L.A., Rider, M.J., Lavorato, M., and PaldilhaFeltrin, A. "An enhanced genetic algorithm to solve the static and multistage transmission network expansion planning", Journal of Electrical and Computer Engineering, 2012 (5) (2012).

28. Silva, I.D.J., Rider, M.J., Romero, R., and Murari, C.A. "Genetic algorithm of Chu and Beasley for static and multistage transmission expansion planning", in Power Engineering Society General Meeting, 2006. IEEE, p. 7 (2006).

29. Silva, I.J., Rider, M.J., Romero, R., and Murari, C.A. "Genetic algorithm of Chu and Beasley for static and multistage transmission expansion planning", in Power Engineering Society General Meeting, 2006. IEEE, p. 7 (2006).

30. 18 bus test system: Data and Results. Available: url\{http://tepstudies.ir/TestSystems/18-bus-system for-snp/\}

31. 51 bus test system Data. Available: url\{http:// tepstudies.ir/TestSystems/iranian-51-bus-system-for$\operatorname{snp} /\}$

\section{Biographies}

Mohammad R. Habibi was born in Baft, Iran in 1988. He received his MSc degree in Electrical Engineering from Kerman Graduate University of Technology (KGUT) at Kerman, Iran in 2011. He is now working for $\mathrm{PhD}$ degree at Electrical Engineering Department of KGUT. His research interests include power system planning, power system optimization, and power system operation.

Masoud Rashidinejad received BSc degree in Electrical Engineering MSc degree in Systems Engineering, from Isfahan University of Technology, Isfahan, Iran, and PhD degree in Electrical Engineering from Brunel University, London, U.K. in 2000. He is currently a Professor with the Department of Electrical Engineering, Shahid Bahonar University of Kerman, Kerman, Iran. His current research interests include power system optimization, power system planning, electricity restructuring, and energy management.

Ali Hajebrahimi received BSc and MSc degrees in Electrical Engineering from Shahid Bahonar University and Kerman Graduate University. $\mathrm{He}$ is currently working toward $\mathrm{PhD}$ degree in Electrical Engineering from Laval University, Quebec, Canada. His research interests include power electronic, management of wind power system, power system reliability, mathematical power system modeling and smart grid.

Amir Abdollahi received BSc degree from Shahid Bahonar University of Kerman, Kerman, Iran in 2007, MSc degree from Sharif University of Technology, Tehran, Iran in 2009, and PhD degree from Tarbiat Modares University, Tehran, Iran in 2012, all in Electrical Engineering. He is currently an Assistant Professor at the Department of Electrical Engineering, Shahid Bahonar University of Kerman, Iran. His research interests include demand-side management, planning, reliability and economics in smart electricity grids. 